\title{
A Congestion Control Algorithm for Mobility Model in Mobile Ad-hoc Networks
}

\author{
Abinasha Mohan Borah \\ Mtech student \\ Assam Don Bosco University
}

\author{
Bobby Sharma \\ Assistant Professor \\ Assam Don Bosco University
}

\author{
Manab Mohan Borah \\ Scientific Officer \\ Indian Institute Of Technology
}

\begin{abstract}
In mobile ad-hoc networks, the mobile nodes are able to move randomly and freely without any centralized administration or control. The mobile nodes in such networks act both as routers and as hosts. The wireless and distributed nature of ad hoc networks implies a major challenge since the chances of losing packets over the network increases to a great extent. Also, packet loss may occur with the increase in size of the data packets which may lead to congestion in the network. In order to detect congestion in the network, various congestion control algorithms are used. This paper gives a brief survey of the various congestion control algorithms used in MANET's and tries to propose a simple congestion control algorithm for mobility models such as random walk model for MANET's. The main objective of this paper is to design an efficient congestion control technique to reduce the amount of congestion in the network.
\end{abstract}

\section{Keywords}

congestion control, MANET, routing, TCP.

\section{INTRODUCTION}

A Mobile Ad-hoc network is a self configuring network of wireless mobile nodes without any centralized control to form an arbitrary topology. The nodes in MANET's are free to move randomly and rapidly from one location to the other without any control. The network's wireless topology is unpredictable and may change rapidly [1]. The fundamental characteristics which differentiate MANETs from other wireless networks is its mobility. Applications in Ad-hoc network are in military communication and operations, emergency services like disaster recovery, commercial and civilian environments [2].

An ad hoc network means arranged or happening whenever necessary and not planned in advance [3]. The nodes in MANET's moves freely in and around the network without any restriction i.e. it has dynamic topology. MANET consists of collection of mobile nodes where each node not only acts as a terminal or host but also acts as a router for forwarding the data from source to destination. Due to the independent behavior of MANET's, there are a number of issues and challenges in designing of MANET networks.

Although MANET's provide communication support where there does not exists any fixed infrastructure, but due to the mobility and presence of limited resources various issues arises that require high research. The issues include security, topology control, quality of service, routing, power management, congestion control etc [4]. The key issue in wireless networks is network congestion and traffic blocking. Congestion occurs in MANET's when the offered load in the network exceeds the available resources resulting in high overhead, packet loss and long delays. If congestion occurs in the network then data will not be transmitted successfully leading to high packet loss. In order to avoid congestion in the network, it is required to use an efficient congestion control algorithm for successful transmission of data throughout the network.

The remainder of the section is organized as follows. Section II gives a brief description of congestion in MANET's and also on the congestion control algorithm. Section III gives a detailed description on the related work done by different researchers on this field. Section IV gives simulation results of our analysis and in section $\mathrm{V}$ we try to propose our congestion control algorithm. And finally section VI gives the conclusion

\section{CONGESTION AND CONGESTION CONTROL ALGORITHM IN MANET'S}

\subsection{Congestion in MANET's}

Congestion can be termed as obstruction where successful communication do not takes place. It is a situation in network communication where too many packets are present in the subnet [5]. The main reason for congestion is the presence of limited resources or when the offered load is greater than the available capacity of the channel. Congestion leads to high packet loss and bandwidth degradation and waste energy and time in its recovery [5]. Congestion can create the following difficulties:

- Long Delay: The congestion control mechanism takes much time for detecting congestion. When the congestion is more severe, it is preferred to select an alternate new path.

- High overhead: It takes into account in discovery of new routes for processing and communication. If multipath routing is used, it needs additional effort for maintaining the multipath regardless of the existence of alternate route.

- Many packet losses: Congestion leads to packet losses. To minimize the traffic load, a congestion control solution is applied either by reducing the sender rate at the sender's side or by dropping the packets at the intermediate nodes or by applying both the methods.

\subsection{Congestion Control in MANET's}

As discussed in previous part, congestion occurs in MANET due to the presence of partial resources and for its recovery a lot of energy and time is wasted. Congestion control method is the technique by which the network bandwidth is distributed across multiple end-to-end connections [6]. Congestion control can be 
rate based or buffer based. Rate based congestion control scheme is usually applied in routing. The main idea for applying any congestion control technique is to increase the throughput, to maximize packet delivery ratio and node transfer in the network and to minimize end-to-end delay, traffic congestion in the network [7].

\section{BACKGROUND STUDY}

Transmission Control Protocol or TCP is the internet protocol that carries $90 \%$ of the internet traffic in both wired and wireless networks. TCP is widely used as a connection-oriented transport layer protocol that provides reliable data transfer between different applications. The main services provided by TCP are reliability, data transfer, flow control, multiplexing, congestion control and connection management [8]. However TCP congestion control may not be suited for MANET's where packet loss may result in invocation of TCP's congestion control mechanisms. In this section we will discuss on TCP variants for congestion control and then we will give an overview on the different congestion control mechanisms in MANET. The reason behind the variations of TCP is that each variant possess a special characteristic. The different variants are given below:

- TCP Tahoe: Traditional TCP or TCP Tahoe was proposed by Van Jacobson was the first TCP variant for congestion control. It is based on the principle of 'conservation of packets' i.e. when the available bandwidth capacity in the network is running out, a packet will not be injected into the network unless a packet is taken out from the network. TCP Tahoe goes through slow start process and congestion window is also maintained. For congestion avoidance it uses Additive Increase Multiplicative Decrease or AIMD technique. The main drawback for this variant is that it takes a complete timeout to detect a packet loss which offers a cost in high bandwidth delay product links [8].

- TCP Reno: Reno has the basic principle of Tahoe of slow start and cross grain but adds an extra feature that the lost packets are detected earlier and the pipeline is not emptied every time a packet is lost. An algorithm called fast retransmit is applied which states that whenever 3 duplicate ACK's are received, it is taken as a sign that the segment was lost and the segment was again retransmitted over the network without waiting for timeout. But this algorithm does not work well for multiple packet losses [10].

- TCP New Reno: New Reno is a modification of Reno in which it is able to detect many packet losses. It is based on basic principle of Reno such as slow start, congestion avoidance and fast re transmit. But it adds an extra feature called fast recovery which allows multiple retransmissions. The problem with this variant is it takes one RTT to detect each packet loss.

- TCP Sack: TCP Sack or TCP Selective Acknowledgement is an extension of TCP Reno. It also has all the features of Reno and Tahoe such as slow start, coarse grained transmission, fast transmit and fast recovery. It works on the problems faced by TCP New Reno namely detection and retransmission of lost packets in one RTT. TCP Sack requires that the segments are not acknowledged cumulatively but to be acknowledged selectively. A new packet is sent if there is no outstanding segment. But the main disadvantage is in the implementation of selective acknowledgement, it is not an easy task [8][9].

The different proposed congestion control algorithms for MANET's are discussed next:

In [11], a technique for MANET's called agent based congestion control for AODV protocol is proposed. In this method the mobile agents MA collects and distributes the information about network congestion. This technique is proposed to avoid the congestion in ad-hoc networks. The main function of the MA is that it starts form a node and moves to its adjacent node from time to time and updates the routing table of the node during its visitation. The node receives the dynamic network topology with the support of the mobile agents. This technique reduces end-to-end delay and tries to attain a high throughput and packet delivery ratio.

In [12], Congestion Adaptive AODV routing protocol CAAODV is proposed whenever multimedia based traffic such as voice, audio, video or text is transmitted over the network. CAAODV is used to address the congestion issues considering routing overhead, delay and packet loss. This protocol ensures the availability of alternative routes along with the primary routes to reduce the routing overhead. If at any point of time, congestion occurs in the primary route between source node and destination node, the concerned node warns its previous node about congestion and an alternate route is selected for transmission to the destination node. This algorithm is concerned for real time communications and is useful for better performance in heavy traffic as well.

In [10], the TCP variants like TCP Reno, TCP New Reno, TCP Sack, TCP Vegas are analyzed, implemented and compared for three different routing protocols. The routing protocols taken in consideration are AODV, DSDV and DSR. The performance metrics like throughput, packet delivery fraction and end-to-end delay are calculated and they found that TCP Vegas is better than any other TCP variant.

Due to the constant movement of nodes in MANET's, energy consumption is higher due to the retransmission of the packets and packet loss. The main objective in this paper is to ensure energy efficiency in the network. In [14] an energy efficient based congestion control scheme (EECCS) is proposed to improve energy consumption and energy efficiency of the mobile nodes. By the simulation results it is found that the proposed scheme achieves minimum energy, low packet loss, low end to end delay, high packet delivery ratio and throughput.

In [13], an agent based congestion control technique is proposed where the information about the network congestion is collected and distributed by the mobile agents. This algorithm was proposed to avoid congestion in the network. The routing protocol used is AODV routing protocol in which the mobile agents moves through the network and updates the routing table according to the node's congestion status. By the simulation results it is observed that this technique attains high throughput and packet delivery ratio with reduced delay and routing load as compared to other existing techniques.

\section{SIMULATION RESULTS}

The simulation study is done by using network simulator NS-2 version 2.35 for mobile ad-hoc networks. Simulator Parameters like Number of nodes, Dimension, Routing protocol, traffic etc are given below: 
Table 1. Simulation Environment with parameters

\begin{tabular}{|c|c|}
\hline NAME & VALUE \\
\hline Simulator Used & Ns- 2.35 \\
\hline Number of nodes & 20,50 \\
\hline Dimension of simulated area & 1000 X 1000 \\
\hline Routing Protocol & AODV \\
\hline Simulation Time & $60 \mathrm{sec}$ \\
\hline Traffic type & TCP \\
\hline Packet size & 1000 bytes \\
\hline Mobility Model & Random walk \\
\hline Pause time & 0 \\
\hline
\end{tabular}

The simulations are performed in $1000 \times 1000$ grid consisting of 20 and 50 mobile nodes in the network. The TCP packet size of 1000 bytes is considered for our analysis. The queue limit is set to 20 packets to avoid frequent drop of packets. The main objective of our analysis is to create a congested network which is done by increasing the number of transmission links and mobility speed. We measured the values of throughput and delay. A network is said to be a congested one if it has higher values for delay and lower values for throughput.

For performance evaluation the following performance metrics are used:

- Throughput: This performance metric represents the total number of bits delivered to the higher layers per second [15].

- Delay: This metric indicates that how long a packet took to travel from the source to the destination.

- Packet delivery ratio: the ratio of the number of packets successfully received to the number of packets transmitted.

- Packet drop ratio: The ratio of occurrence of packet lost to the total number of packets used in the network during one transmission phase is defined as packet loss rate.

Below we present some of our simulation results:

Figure 2 shows the traffic creation along with the mobility of the nodes. The number of nodes created is 50 and the communication links is 35 . The delay is produced from source to destination via neighbor nodes.

Figure 3 and figure 5 shows the result of throughput for 20 and 50 mobile nodes. The mobility speed is taken to be $25 \mathrm{~m} / \mathrm{s}$ for varying communication links 5, 10, 20 and 35. From the results, we can see that throughput gradually decreases as we increase the number of communication links in the network. A decrease in throughput in the network indicates that there is congestion in the network and it needs to be controlled for successful delivery of the messages.

Figure 4 and figure 6 shows the result of throughput for 20 and 50 mobile nodes with varying mobility speed. The mobility speed taken for analysis is $25,35,45$ and $55 \mathrm{~m} / \mathrm{s}$ with 10 communication links. From the results, it is seen that throughput decreases as we increase mobility speed. An increase in mobility speed degrades the performance of the network.
Figure 7 shows an increase in delay with respect to the number of communication links in the network. For better performance it is necessary to have minimum values for delay and maximum values of throughput.

From the above analysis we found that by increasing the parameters like communication link and mobility speed we find that there is a change in the performance metrics. The metric throughput decreases when we go on increasing the mobility speed and communication links in the network and delay for the network gradually increases. The above analysis shows that our network is a congested network and it needs to be controlled. In the next section we propose an algorithm for congestion control for mobility models. This model will try to decrease the congestion in the network.

\section{PROPOSED MODEL}

Figure 7 represents the flowchart of ou congestion control algorithm where RWM and LWM are the mobility models i.e. random walk mobility model and levy walk mobility model. Pdr is the packet drop ratio and $\mathrm{pdr}_{\text {thr }}$ is the threshold value for packet drop ratio. The threshold values can be obtained by calculating the average values for packet drop ratio. If the values for pdr, delay goes on increasing above the threshold level and the value for throughput decreases below threshold level then we will consider that our network is congested, if not then it will be a stable one. If the network is congested it will try to calculate the buffer space, load and available channel capacity. If these values are below the normal rate, then the intermediate nodes will send a message to the sender to send less number of packets and then it will calculate the congestion in the network. If congestion is controlled the process will stop, else we will assume that they are caused by other types of attacks.

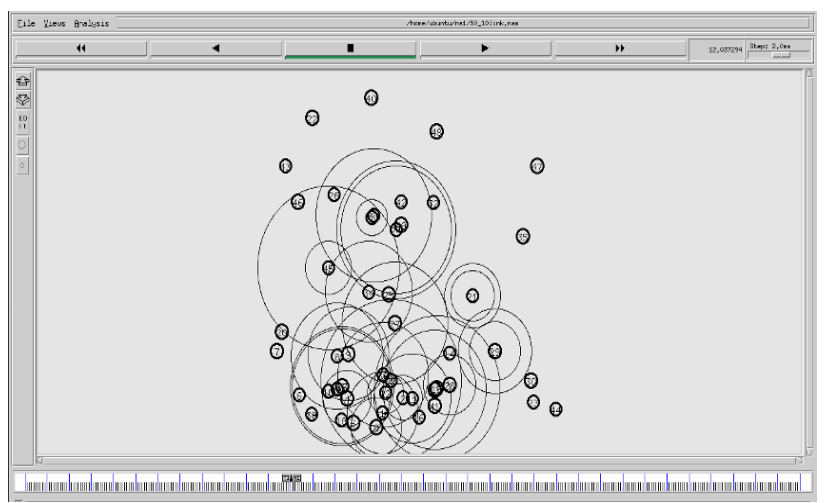

Figure 1: Topology and Traffic Connection of Random Walk Model

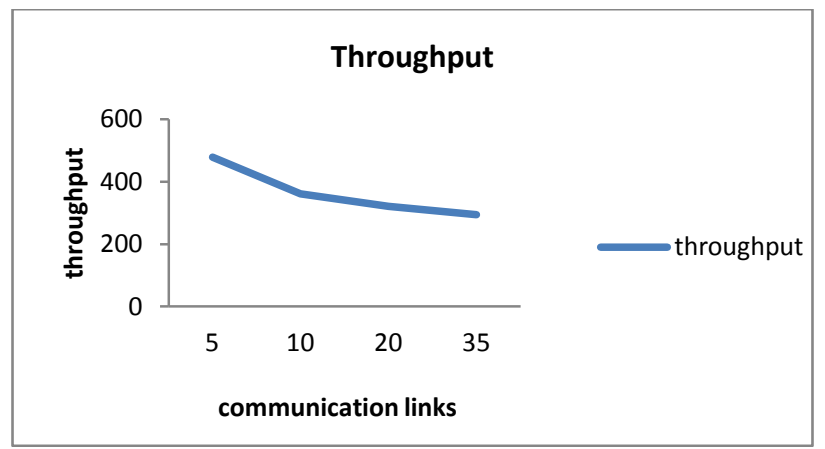

Figure 2: Throughput vs Congestion graph w.r.t number of communication links for 20 mobile nodes 


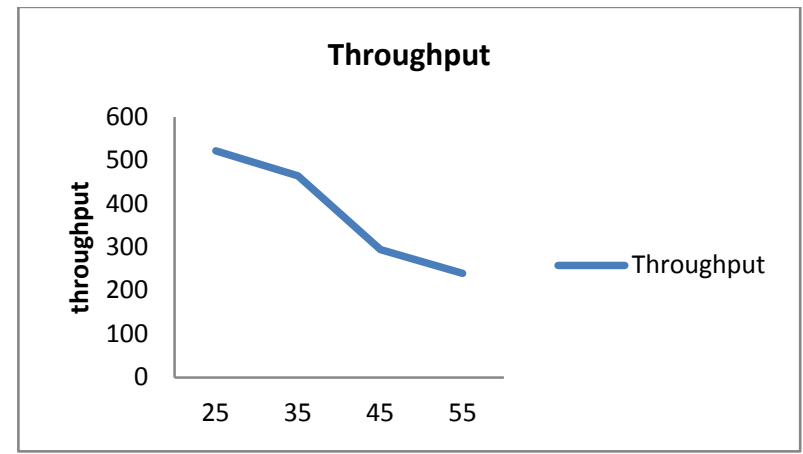

Figure 3: Throughput vs Congestion graph w.r.t mobility speed for $20 \mathrm{MN}$

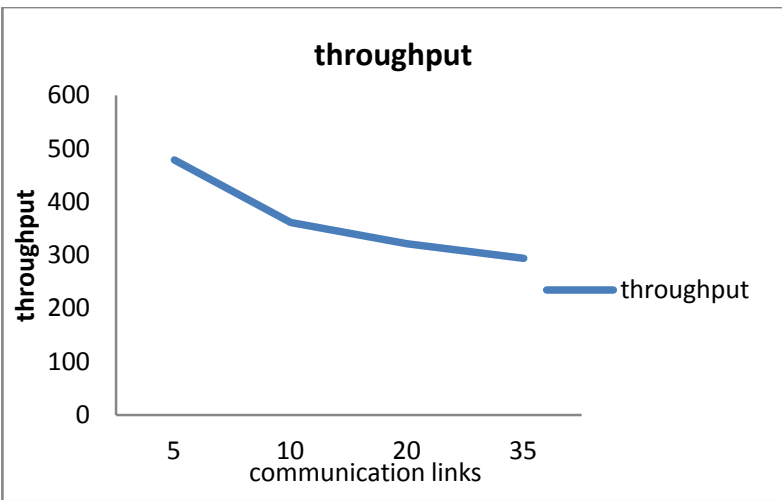

Figure 4: Throughput vs Congestion graph w.r.t number of communication links for $\mathbf{5 0}$ mobile nodes

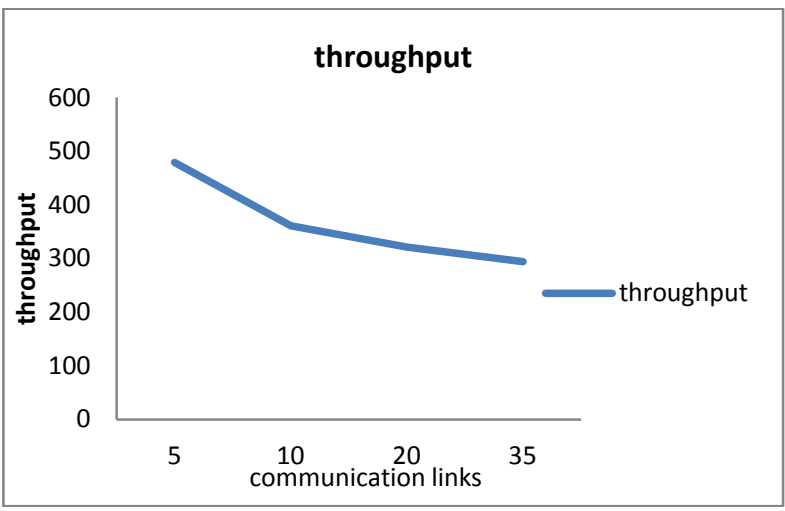

Figure 5: Throughput vs Congestion graph w.r.t mobility speed for 50 mobile nodes

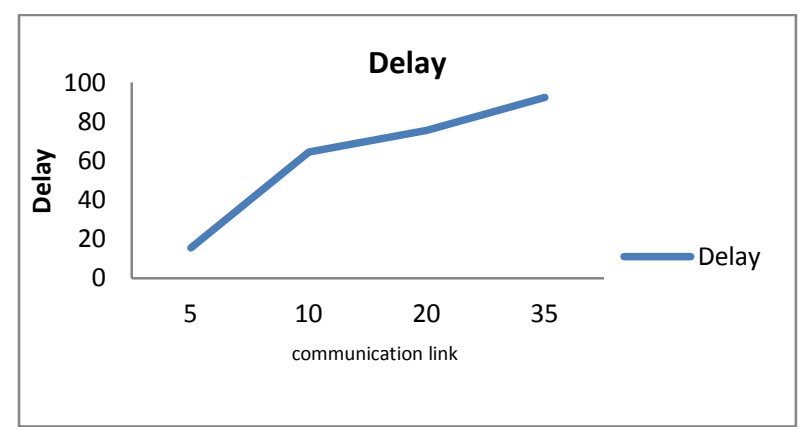

Figure 6: Delay vs Congestion graph w.r.t number of communication links for $\mathbf{5 0}$ mobile nodes.

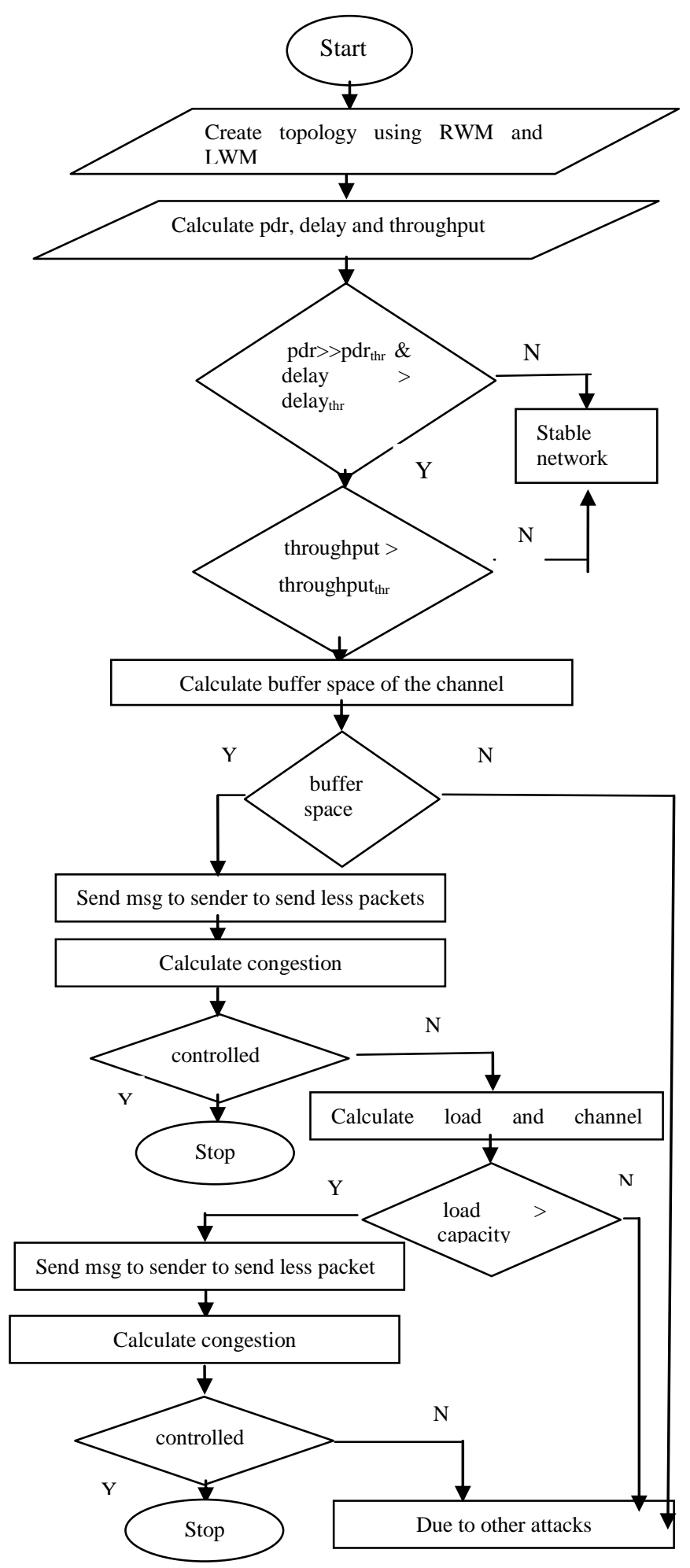

Figure 7: Our Proposed Flowchart for congestion control 


\section{REFERENCES}

[1] Ivan Stojmenovic, 2002 "Mobile Ad-hoc Networks and Routing Protocols," in Handbook of Wireless Networks and Mobile Computing, 2nd ed. John Wiley \& Sons

[2] Jeroen Hoebeke, Ingrid Moerman, Bart Dhoedt, Piet Demeester. An Overview of Mobile Ad-hoc Network: Applications and Challenges. IEEE.

[3] Fan Bai, Ahmed Helmy. A Survey of Mobility Models in Wireless Ad-hoc Networks. University of Southern California.

[4] Tracy Camp, Jeff Boleng, Vanessa Davies, 2002. A Survey of Mobility Models for Ad-hoc Network Research. IEEE

[5] Barkha Shakya, Deepak Kulhare, Arpit Solanki, 2013. Investigation of TCP Congestion Controlwith Reliable Communication Techniques for MANET . International Journal of Computer Applications Vol 65 No-14. Pp 09758887.

[6] H. Gupta, 2013. Survey of Routing based Congestion control Techniques under MANET. In International Conference on Emerging Trends in Computing, Communication and Nanotechnology.

[7] Parminder Kaur, Ranjit Singh, 2013. A systematic Approach for Congestion Control in Wireless Ad-hoc Networks. International Journal of Advanced Research in Computer and Communication Engineering, Vol 2 Issue 3.
[8] Madiha Kazmi, Muhammad Javed, 2011. An overview of Performance Comparisons of Different TCP Variants in IP Networks. Network Digital Technologies Communications in Computer and Information Science.

[9] K. Fall, S. Floyd. Simulation Based Comparison of Tahoe, Reno and Sack TCP.

[10] Jitender Sharma, Hardeep Singh, 2012. Analysis of Reno: A TCP Variant. International Journal of Electronics and Communication Engineering.

[11] Vishnu Kumar Sharma, Dr Sarita Singh Bhadauria, 2012. Agent Based Congestion Control Using AODV Routing Protocol Technique for Mobile Ad-hoc Network. International Journal of Wireless and Mobile Networks.

[12] Boraiah Ramesh, 2008. CA-AODV Congestion Adaptive AODV Routing Protocol for Streaming Video in Mobile Ad-hoc Networks. I.J Communications, Network and System Sciences.

[13] Bandana Bhatia, Neha Sood, 2014. AODV Based Congestion Control Protocols. International Journal of Computer Science and Information Technology.

[14] Zequn Huang, Jaeil Jung. An Energy Efficient Congestion Control Technique for Wireless Sensor Networks. International Journal Of Computer Applications. 